\title{
The Female Population Growth Projection Year 2021 in Trenggalek Regency by Leslie Matrix Model on the Birth Rate and Life Expectancy
}

\author{
Dewi Anggreini \\ Mathematics Education Department STKIP PGRI Tulungagung, \\ Jl. Mayor Sujadi East Street, No.7, Tulungagung, 66221, Indonesia \\ Author correspondency: \\ anggreini_004@yahoo.com
}

\begin{abstract}
This research aims to determine the number of female residents in Trenggalek Regency in 2021 based on data on birth rate and life expectancy. The use of eigenvalues and eigenvectors aims to determine the dividing age distribution by Leslie matrix model. The eigenvectors are used to determine the number of female populations of each age interval, while the eigenvalues are used to determine population growth rates. The research method used is to determine the subject of research. The next stage is to collect research data, then analyze the data and last draw conclusions. The research data is obtained from BPS Kabupaten Trenggalek and BPS East Java Province that is data of woman population from year 2010-2015. The result of this research using Leslie matrix model for female population in Trenggalek Regency that is discrete model. The discrete model is divided into fourteen age intervals constructed using the birthrate and life expectancy. The conclusions of the study showed that the number of female population in Trenggalek Regency tended to increase with positive eigen value greater than one. In other words, the growth rate of female population in Trenggalek Regency tends to be positive. The success of Leslie's matrix model is the application of case studies in predicting the number of female populations in Trenggalek District by 2021 using the MAPLE 16 Program.
\end{abstract}

Keywords: Eigen value; eigenvector; leslie matrix.

\section{INTRODUCTION}

Trenggalek Regency is a Regency in East Java province which is located in the southern part of the province of East Java with an area of $1,261.40 \mathrm{~km}^{2}$. Trenggalek Regency largely made up of mountainous land with an area of $2 / 3$ covers part of the area. While the rest of his $(1 / 3$ part $)$ is the land of the lowlands. Trenggalek Regency is subdivided into 14 Subdistricts da 157 villages. Only about 4 subdistricts which are the majority of the village of plains, which are: Pogalan, Trenggalek Subdistrict, district and Sub-district Durenan Monument. While the majority of other Sub 10 village mountains. A large number of Trenggalek Regency Year 2015 as much 689,200 inhabitants.

Number of changes in the populations affected by the internal state of the population is birth, death, and survival. The existence of a number of changes of a population called the growth of population. Population growth can provide information as to whether changes in population numbers for next year is always increasing, decreasing or constant. (Pratama, Prihandono and Kusumastuti, 2013, p. 163).

Population projection is not a forecast but a population of scientific calculations based on the assumption of the components of population growth rate, i.e., birth, death, and displacement. The third component is what determines the magnitude of the age structure of the population and the population in the future. To determine each assumption needed data that illustrates the trend in the past to the present, factors that affect the relationship between a component and a component with another as well as the expected target is reached on the future come.

Science many rapidly developing lately, including mathematical modeling. According to Iswanto (2012, p. 19) in its development of mathematical models can be represented on the problems that occur in everyday life. So there is a strong relationship between applied science and mathematics are represented by mathematical models that are designed and implemented with the help of computer science, to facilitate the simulation of realworld systems. Population growth is one of the examples of the application of the Linear Algebra in field biology and Ecology specifically quantitatively. Population growth in ecology is often referred to as the "population dynamics". Ecology is usually defined as the relationship between living beings with their environment (Tarumingkeng, 1994, p. 6).

Many models that can be used to explain the growth of the population. One of the models used by demographers is the model Leslie. Where the model using a mathematical approach, i.e. the matrix. In the model Leslie that birth and death processes depending on the age and become an important part in the growth of the population. In General, the growth of a living 
creature is a process of continuous or ongoing process taking place. However, the study population should be also approached from a discrete time review. The use of discrete pattern based also upon the observations of the population that is generally done interval-the interval period such as a day, a week, and the units of time according to a draft of the researchers concerned. Based on these considerations, in addition to growth models based on the continuous solutions need to be evaluated more thoroughly with the solution of the discrete basis. (Tarumingkeng, 1994, p. 27). In addition, the existence of significant size have yet to know the growth of female population in Trenggalek based on birth rate and life expectancy for the coming year as well as the limiting distribution of unknown age by using model the Leslie matrix.

There is some research on Matrix Leslie i.e. research Corazon, Nurul, h. and Yusienta, m., (2016, p. 6) which uses a matrix of Leslie to predict the number and pace of growth in the province of Riau in the year 2017. So the obtained results of the total population of women in Riau Province tend to experience increased. In addition the results of research conducted by Pratama, Prihandon. From the description it is necessary to determine the abundance of female population and knowing the limiting age distribution of the female population in Trenggalek based on birth rate and life expectancy using the eigen values and eigen vectors matrix of Leslie.

\section{MATERIALS AND METHODS}

This research is research with quantitative approach with the types of descriptive research. This research is done by taking a secondary data in some of the Central Bureau of statistics in East Java province and Trenggalek Regency BPS. The sample in this study is a population of women of the years 2010 to 2015, by comparison with the number of child birth from the year 2010 to the year 2015. From the results of the data then diplikasikan by applying the matrix model Leslie to look for population, the value of the eigenvalues and eigen vectors.

\section{Procedure Research}

The research methods used in this research are: the first stage is to determine the subject of the research, as for the subject of his research is the female population on the birth rate and life expectancy in East Java province and the second stage is to (1) collect research data, with regard to the collection of research data obtained from secondary data in the Central Bureau of statistics of East Java province and Trenggalek Regency BPS (2) data analysis and the last is an interesting conclusion.

\section{Observed Variables}

Observed variables in this study was the value of eigen and eigen vectors of the matrix Leslie. Eigen values of L are the roots of polynomials of its characteristics. The observed variables in this study was the value of eigen and eigen vectors of the matrix Leslie. Eigen values of $\mathrm{L}$ are the roots of polynomials of its characteristics. Polynomial matrix characteristics of Leslie are:

$$
\begin{aligned}
& p(\lambda)=\lambda^{n}-a_{1} \lambda^{n-1}-a_{2} b_{1} \lambda^{n-2}-a_{3} b_{1} b_{2} \lambda^{n-3} \cdots- \\
& a_{n} b_{1} b_{2} \cdots b_{n-1} \\
& \mathbf{x}=\left[\begin{array}{c}
x_{1} \\
x_{2} \\
x_{3} \\
\vdots \\
x_{n}
\end{array}\right]
\end{aligned}
$$

A vector of eigenvalues of a Leslie Matrix $L$ related $\lambda_{1}$ If and only if $\mathrm{x}$ is a non-trivial solution of $(\lambda I-$ $L) \mathbf{x}=0$.

\section{Data Analysis Technique}

In doing data analysis techniques after the data is finished being processed which do first is find the values of ai (a fertility figure female population) obtained from the quotient between the average number of births born son a mom on 2010-2015 year divided by the number of female population in the year 2010 and bi (life expectancy female population) are obtained from the results of the women's Division of the population the year 2015 with total population of woman of the year 2010. Second, the mengkontruksi model matrix of Leslie on the growth of the population. Third after Matric Leslie was formed later enter the data the number of female population in 2015 to generate predictions of the total population of women in the year 2021. Third, find the value of a positive eigenvalues in the matrix. Fifth, determine the value of the eigen vectors eigen were positive. Sixth, using equation approach to determine the limiting age distribution. The seventh note the abundance of female population for a long period to come. The eighth research report compiled and processed data through the application of MAPLE 16.

\section{Matrix}

According to Kariadinata (2013, p.11) Matrix is the arrangement of a group of numbers in a rectangular shaped ranks arranged based on row and column, and placed between two brackets. Meanwhile, according to Anton, H. \& Rorres, C. (2004, p. 23) a matrix with $n$ rows and $n$ columns is called an $n$ order square matrix (in square matrices of order $\mathrm{n}$ ), and the entries $a_{11}, a_{22}, \ldots, a_{n n}$ on the main diagonal is said to be from a. When $\mathrm{A}$ is the matrix has $\mathrm{n}$ rows and $\mathrm{n}$ column (type $\mathrm{n} \times \mathrm{n}$ ), then $\mathrm{A}$ can be written as:

$\left[\begin{array}{cccc}a_{11} & a_{12} & \cdots & a_{1 n} \\ a_{21} & a_{22} & \cdots & a_{2 n} \\ \vdots & \vdots & & \vdots \\ a_{n 1} & a_{n 2} & \cdots & a_{n n}\end{array}\right]$ 


\section{Inverse of a Matrix}

According to Adiwijaya (2014, p. 10) Suppose $A$ and $B$ are square matrices of the same size are and $I$ is the identity matrix. If $A \cdot B=I$ then $B$ is called the inverse of matrix A (on the contrary, A is the inverse of matrix $\mathrm{B}$ ). Notation that the inverse of matrix $\mathrm{B}$ is $\mathrm{A}$ is $\mathrm{B}=A^{-1}$ and vice versa $A=B^{-1}$

\section{Matriks Elementer}

According to Anton, H \& Rorres, C (2004, p.56) An $n$ x $n$ matrix is called elementary matrix if the matrix can be derived from $I_{n} n \times n$ identity matrix by performing a single elementary row operation. Each elementary matrix can be reversed, and its inverse is also an elementary matrix. If $A$ is an $n \times n$, matrix, then the following statements are equivalent,they are all true or all wrong: a) A is invertible, b) $A x=0$ has only a trivial solution, $c$ ) A row equivalent to $I_{n}$ or reduced rowechelon form $A$ is $I_{n}$.

\section{Determinants}

According to Anton, $\mathrm{H} \&$ Rorres, C (2004, p.92) an elementary product of a matrix $\mathrm{A}, A, n \times n$, is a product of entries of, none of which come from the same row or column.

\section{Eigenvalues and Eigenvectors}

According to Kariadinata (2013, p.209) if $A$ is an $n \times n$ size matrix, then the nonzero vector $\mathbf{x}$ in $R^{n}$ is called an eigenvector of $A$ if $A \mathbf{x}$ is a scalar multiple of $\mathbf{x}$; ie, $A \boldsymbol{x}=$ $\lambda \boldsymbol{x}$ for any scalar $\lambda$. The scalar $\lambda$ is called the eigenvalue of $A$ and $\mathbf{x}$ is said to be the eigenvector corresponding to $\lambda$. To find the eigenvalue of the matrix $A$ of $n \times n$ size then $A \boldsymbol{x}=\lambda \boldsymbol{x}$ can be rewritten as $A \boldsymbol{x}=\lambda I \boldsymbol{x}$ or equivalent to $(\lambda I-A) \mathbf{x}=0$. To be an eigenvalue, there must be a nonzero solution of the equation $(\lambda I-A) \mathbf{x}=$ 0 , obtained if and only if $\operatorname{det}(\lambda I-A) \mathbf{x}=0$.

The equation of $\operatorname{det}(\lambda I-A) \mathbf{x}=0$ is called the characteristic equation of $A$; the scalar that satisfies this equation is the eigenvalue of $\mathrm{A}$. When expanded, then $\operatorname{det}(\lambda I-A)$ is a polynomial $p$ in a variable called characteristic polynomial of $A$. The characteristic polynomial $p^{(x)}$ of an $n \times n$ matrix has the form:

$p(\lambda)=\operatorname{det}(\lambda I-A)=\lambda^{n}+C_{1} \lambda^{n-1}+\cdots+C_{n}$

\section{Diagonalization of the Matrix}

According to Anton, H \& Rorres, C (2004, p.74) the diagonal matrix of a square matrix whose entry does not lie in the main diagonal is zero is called the diagonal matrix. A common diagonal matrix $D, n \times n$ can be written

$\left[\begin{array}{cccc}d_{1} & 0 & \cdots & 0 \\ 0 & d_{2} & \cdots & 0 \\ \vdots & \vdots & & \vdots \\ 0 & 0 & \cdots & d_{n}\end{array}\right]$
A diagonal matrix can be reversed, if and only if all entries in the main diagonal position are non-zero; in this case the inverse of $\mathrm{D}$ is

$D^{-1}=\left[\begin{array}{cccc}1 / d_{1} & 0 & \cdots & 0 \\ 0 & 1 / d_{2} & \cdots & 0 \\ \vdots & \vdots & & \vdots \\ 0 & 0 & \cdots & 1 / d_{n}\end{array}\right]$

So, $D D^{-1}=D^{-1} D=I$.

\section{RESULTS AND DISCUSSION}

\section{Leslie Matrix Model in Population Growth}

In Leslie's model, women or females are divided over age groups that are timed together. Specifically, uppose that the maximum age reached by any woman or female in the population is $M$ years (or expressed in other units of time) and then the population is divided over $n$ the age group. So the time period in each group $M / n$ years. The age group will be explained by the following table:

Table 1. Age Group of Leslie Matrix Model.

\begin{tabular}{ll}
\hline Age Group & Age Interval \\
\hline \hline 1 & {$[0, M / n]$} \\
2 & {$[M / n, 2 M / n]$} \\
3 & {$[2 M / n, 3 M / n]$} \\
$:$ & $:$ \\
$:$ & $:$ \\
$(\mathrm{n}-1)$ & {$[(\mathrm{n}-2) 2 M / n,(\mathrm{n}-1) M / n]$} \\
$\mathrm{n}$ & {$[(\mathrm{n}-1) M / n, M]$} \\
\hline
\end{tabular}

For example known to the number of women or females in each group of to- $n$ group at time $t=0$. Specifically, for example, there are $x_{1}^{(0)}$ women or females in the first group, $x_{2}{ }^{(0)}$ woman or female in second group, $x_{3}{ }^{(0)}$ woman or female in the third group, and so on. With the number ke- $n$ will be formed a column vector $\mathbf{x}^{(0)}$ that is:

$\mathbf{x}^{(0)}=\left[\begin{array}{c}x_{1}{ }^{(0)} \\ x_{2}{ }^{(0)} \\ \vdots \\ x^{(0)}\end{array}\right]$

This vector is called the age distribution vector of the origin (initial age distribution vector). Over time, the number of women or females in each group of to $-n$ the group will change due to three biological processes, namely: birth, death and aging. By explaining these three processes quantitatively, it can be seen how to project the vector of early age distribution into the future. The easiest way to learn about the aging process is to observe the population at discrete times, say $t_{0}, t_{1}, \ldots, t_{k}, \ldots$ Leslie's model requires that the time span between two consecutive observation times is the 
same as the time period of the hose (interval) age, and is written as follows:

$t_{0}=0, t_{1}=M / n, t_{2}=2 M / n$, to $t_{k}=k M / n$

With this assumption, all women or females in the group- $(i+1)$ at $t_{k+1}$ time have been in group to- $i$ at time $t_{k}$. The process of birth and death processes between two consecutive observation times can be explained using demographic parameters. As follows:

Table 2. Parameters in Leslie Matrix Model.

\begin{tabular}{cl}
\hline Parameter Model & Information \\
\hline$a_{i}$ & Average number of girls \\
$i=1,2, \ldots, n$ & who was born by a woman during her \\
& time in the age group i. \\
$b_{i}$ & The number of women in the i-age \\
$i=1,2, \ldots, n$ & group who can be expected is still \\
& alive and up to age group i. \\
\hline
\end{tabular}

By defition, it will be obtained that (i) $a_{i} \geq 0$ for $i=1,2, \ldots, n$ and (ii) $0<b_{i} \leq 1$ for $i=1,2, \ldots, n-$
1.Can be Seen that, should not allow for $b_{i}$ which is equal to zero, because if this happens then there will be no female or female alive after the age group to- $i$. And also considered that at least there is one $a_{i}$ positive that will occur birth. Each age group in which the corresponding value $a_{i}$ is positive is called the fertile age group (fertile age class). Next we will define the age distribution vector $x^{(k)}$ at the time $t_{k}$ with:

$\mathbf{x}^{(k)}=\left[\begin{array}{c}x_{1}{ }^{(k)} \\ x_{2}{ }^{(k)} \\ \vdots \\ x^{(k)}\end{array}\right]$

Where $x_{i}^{(k)}$ is the number of women or females in the i-age group at the time $t_{k}$. At times, women in the first age group are daughters of women born between time $t_{k-1}$ and time $t_{k}$. So, it can be written

$$
\left(\begin{array}{l}
\text { Number of } \\
\text { women in group } \\
1 \text { at time } t_{k}
\end{array}\right)=\left(\begin{array}{l}
\text { Number of daughters born } \\
\text { by women in group 1 } \\
\text { between } t_{k-1} \text { and time } t_{k}
\end{array}\right)+\left(\begin{array}{l}
\text { Number of daughters born } \\
\text { by women in group 2 } \\
\text { between } t_{k-1} \text { and time } t_{k}
\end{array}\right)+\ldots+\left(\begin{array}{l}
\text { Number of daughters born } \\
\text { by women in group } \mathrm{n} \\
\text { between } t_{k-1} \text { and time } t_{k}
\end{array}\right)
$$

Or mathematically,

$x_{1}{ }^{(k)}=a_{1} x_{1}{ }^{(k-1)}+a_{2} x_{2}{ }^{(k-1)}+\cdots+a_{n} x_{n}{ }^{(k-1)}$

Number of women in the age group to $-(i+$ 1) $(i=1,2, \ldots, n-1)$ at the time $t_{k}$ are women in groups to $-i$ at the time $t_{k-1}$ who are still alive at the time $t_{k}$. Or mathematically

$x_{i+1}^{(k)}=b_{i} x_{i}^{(k-1)}, i=1,2, \ldots, n-1$

By using matrix notation, equations (1) and (2) can be written in the form

$$
\left[\begin{array}{c}
x_{1}{ }^{(k)} \\
x_{2}{ }^{(k)} \\
x_{3}{ }^{(k)} \\
\vdots \\
x_{n}{ }^{(k)}
\end{array}\right]=\left[\begin{array}{cccccc}
a_{1} & a_{2} & a_{3} & \ldots & a_{n-1} & a_{n} \\
b_{1} & 0 & 0 & \ldots & 0 & 0 \\
0 & b_{2} & 0 & \cdots & 0 & 0 \\
\vdots & \vdots & \vdots & \vdots & \vdots & \vdots \\
0 & 0 & 0 & 0 & b_{n-1} & 0
\end{array}\right]\left[\begin{array}{c}
x_{1}{ }^{(k-1)} \\
x_{2}{ }^{(k-1)} \\
x_{3}{ }^{(k-1)} \\
\vdots \\
x_{n}{ }^{(k-1)}
\end{array}\right]
$$

Or more succinctly

$\mathbf{x}^{(k)}=L \mathbf{x}^{(k-1)}$, with $k=1,2, \ldots$
Where $L$ is Leslie Matrix

$L=\left[\begin{array}{ccccccc}a_{1} & a_{2} & a_{3} & \ldots & a_{n-1} & a_{n} \\ b_{1} & 0 & 0 & \ldots & 0 & 0 \\ 0 & b_{2} & 0 & \cdots & 0 & 0 \\ \vdots & \vdots & \vdots & \vdots & \vdots & \vdots \\ 0 & 0 & 0 & 0 & b_{n-1} & 0\end{array}\right]$

From the equation (3) is found that

$$
\begin{aligned}
& \mathbf{x}^{(1)}=L \mathbf{x}^{(1)} \\
& \mathbf{x}^{(2)}=L \mathbf{x}^{(1)}=L^{(2)} \mathbf{x}^{(0)} \\
& \mathbf{x}^{(3)}=L \mathbf{x}^{(2)}=L^{(3)} \mathbf{x}^{(0)} \\
& \vdots \\
& \mathbf{x}^{(k)}=L \mathbf{x}^{(k-1)}=L^{(k)} \mathbf{x}^{(0)}
\end{aligned}
$$

Thus, if we know the start age distribution $\mathbf{x}^{(0)}$ and Leslie Matrix $L$, then we can determine the age distribution of women or females at any later time.

\section{Limited Age Distribution}

Although the equation (5) gives the age distribution of the population at any given time, it does not immediately provide an overview of the dynamics of the 
growth process. It is necessary to investigate the eigenvalues and eigenvectors of Leslie's matrix. The eigenvalues of $L$ are the roots of the characteristic polynomial. The characteristic polynomial of Leslie's matrix is

$$
\begin{aligned}
& p(\lambda)=\lambda^{n}-a_{1} \lambda^{n-1}-a_{2} b_{1} \lambda^{n-2}-a_{3} b_{1} b_{2} \lambda^{n-3} \cdots- \\
& a_{n} b_{1} b_{2} \cdots b_{n-1}
\end{aligned}
$$

Will be given the theorems related to Leslie's matrix, as follows:

\section{Theorem 1}

A Leslie Matrix L has a unique positive eigen value $\lambda_{1}$. This eigenvalue has a multiplicity of 1 and has an eigenvector $\mathbf{x}_{1}$ that all entries are positive.

\section{Evidence:}

(i) The Leslie matrix $L$ has a positive eigenvalue $\lambda_{1}$.

The eigenvalue is the root of the characteristic equation, that is

$$
\begin{aligned}
& p(\lambda)=|\lambda I-L|=0 \\
& p(\lambda)=\lambda^{n}-a_{1} \lambda^{n-1}-a_{2} b_{1} \lambda^{n-2}-a_{3} b_{1} b_{2} \lambda^{n-3} \\
& \cdots-a_{n} b_{1} b_{2} \cdots b_{n-1}
\end{aligned}
$$

If $p(\lambda)=0$, so

$$
\begin{aligned}
& \lambda^{n}-a_{1} \lambda^{n-1}-a_{2} b_{1} \lambda^{n-2}-a_{3} b_{1} b_{2} \lambda^{n-3} \\
& \cdots-a_{n} b_{1} b_{2} \cdots b_{n-1}=0
\end{aligned}
$$

Because one of the roots of the equation (6) must be the dividing factor of the coefficient

$a_{n} b_{1} b_{2} \ldots b_{n-1}$, i.e $\pm a_{n}, \pm b_{1}, \pm b_{2}, \cdots, \pm b_{n-1}$

Suppose that the solution of one of the divider factors, for example, is taken $b_{1}>0$ as a solution or $b_{1}$ as its root, so the equation (6) becomes

$$
\begin{aligned}
& p(\lambda)=\lambda^{n}-a_{1} \lambda^{n-1}-a_{2} b_{1} \lambda^{n-2}-a_{3} b_{1} b_{2} \lambda^{n-3} \\
& \cdots-a_{n} b_{1} b_{2} \cdots b_{n-1}=0 \\
& \lambda^{n}=a_{1} \lambda^{n-1}+a_{2} b_{1} \lambda^{n-2}+a_{3} b_{1} b_{2} \lambda^{n-3} \\
& \cdots+a_{n} b_{1} b_{2} \cdots b_{n-1} \\
& \left(\lambda-b_{1}\right)\left(a_{1} \lambda^{n-1}+a_{2} b_{1} \lambda^{n-2}+a_{3} b_{1} b_{2} \lambda^{n-3}+\cdots\right. \\
& \left.+a_{n} b_{2} \cdots b_{n-1}\right)=\lambda^{n}
\end{aligned}
$$

Means obtained a positive eigen value $\lambda_{1}$, that is $\lambda_{1}=b_{1}$. It will then be proven that Leslie matrix $L$ has a $\lambda_{1}$ single eigenvalue that single, that is $\lambda_{1}=b_{1}$. It will be assumed that Leslie's matrix has another positive eigenvalue. So to prove it used a way of contradiction. An example $\lambda_{2}$ is another positive eigenvalue assuming $\lambda_{1} \neq \lambda_{2}$ or $\lambda_{2} \neq b_{1}$.
Is known

$$
\begin{aligned}
& p(\lambda)=\lambda^{n}-a_{1} \lambda^{n-1}-a_{2} b_{1} \lambda^{n-2}-a_{3} b_{1} b_{2} \lambda^{n-3} \\
& \cdots-a_{n} b_{1} b_{2} \cdots b_{n-1}
\end{aligned}
$$

If $p(\lambda)=0$, then,

$\lambda^{n}-a_{1} \lambda^{n-1}-a_{2} b_{1} \lambda^{n-2}-a_{3} b_{1} b_{2} \lambda^{n-3}$

$\cdots-a_{n} b_{1} b_{2} \cdots b_{n-1}=0$

The two segments are divided by $\lambda^{n}$, be

$$
1=\frac{a_{1}}{\lambda}+\frac{a_{2} b_{1}}{\lambda^{2}}+\frac{a_{3} b_{1} b_{2}}{\lambda^{3}}+\cdots+\frac{a_{n} b_{1} b_{2} \ldots b_{n-1}}{\lambda^{n}}
$$

So $q(\lambda)=1$, for $\lambda \neq 0$ or

$q(\lambda)=\frac{a_{1}}{\lambda}+\frac{a_{2} b_{1}}{\lambda^{2}}+\frac{a_{3} b_{1} b_{2}}{\lambda^{3}}+\cdots+\frac{a_{n} b_{1} b_{2} \cdots b_{n-1}}{\lambda^{n}}$

Proven that $\lambda_{1}$ and $\lambda_{2}$ is the solution of the system of equations

$q(\lambda)=1$. So get it $q\left(\lambda_{1}\right)=1$ and $q\left(\lambda_{2}\right)=1$ or $q\left(\lambda_{1}\right)=q\left(\lambda_{2}\right)$.

Then by using the equation (7), and if $q\left(\lambda_{1}\right)=q\left(\lambda_{2}\right)$

$$
\begin{aligned}
& \frac{a_{1}}{\lambda_{1}}+\frac{a_{2} b_{1}}{\lambda_{1}{ }^{2}}+\frac{a_{3} b_{1} b_{2}}{\lambda_{1}{ }^{3}}+\cdots+\frac{a_{n} b_{1} b_{2} \ldots b_{n-1}}{\lambda_{1}{ }^{n}}= \\
& \frac{a_{1}}{\lambda_{2}}+\frac{a_{2} b_{1}}{\lambda_{2}{ }^{2}}+\frac{a_{3} b_{1} b_{2}}{\lambda_{2}{ }^{3}}+\cdots+\frac{a_{n} b_{1} b_{2} \ldots b_{n-1}}{\lambda_{2}{ }^{n}}
\end{aligned}
$$

If $q\left(\lambda_{1}\right)$ deducted by $q\left(\lambda_{2}\right)$ then

$a_{1}\left(\frac{1}{\lambda_{1}}-\frac{1}{\lambda_{2}}\right)+a_{2} b_{1}\left(\frac{1}{\lambda_{1}{ }^{2}}-\frac{1}{\lambda_{2}^{2}}\right)+a_{2} b_{1} b_{2}\left(\frac{1}{\lambda_{1}^{3}}-\frac{1}{\lambda_{2}{ }^{3}}\right)+$ $\ldots+a_{n} b_{1} b_{2} \ldots b_{n-1}\left(\frac{1}{\lambda_{1}{ }^{n}}-\frac{1}{\lambda_{2}{ }^{n}}\right)=0$

$\mathrm{Be}$

$a_{1}\left(\frac{\lambda_{2}-\lambda_{1}}{\lambda_{1} \lambda_{2}}\right)+a_{2} b_{1}\left(\frac{\lambda_{2}{ }^{2}-\lambda_{1}{ }^{2}}{\lambda_{1}{ }^{2} \lambda_{2}{ }^{2}}\right)+a_{2} b_{1} b_{2}\left(\frac{\lambda_{2}{ }^{3}-\lambda_{1}{ }^{3}}{\lambda_{1}{ }^{3} \lambda_{2}{ }^{3}}\right)+$ $\ldots+a_{n} b_{1} b_{2} \ldots b_{n-1}\left(\frac{\lambda_{2}{ }^{n}-\lambda_{1}{ }^{n}}{\lambda_{1}{ }^{n} \lambda_{2}{ }^{n}}\right)=0$

or

$$
\begin{aligned}
& \frac{a_{1}}{\lambda_{1} \lambda_{2}}\left(\lambda_{2}-\lambda_{1}\right)+\frac{a_{2} b_{1}}{\lambda_{1}{ }^{2} \lambda_{2}{ }^{2}}\left(\lambda_{2}{ }^{2}-\lambda_{1}{ }^{2}\right)+\frac{a_{2} b_{1} b_{2}}{\lambda_{1}{ }^{3} \lambda_{2}{ }^{3}}\left(\lambda_{2}{ }^{3}-\lambda_{1}{ }^{3}\right) \\
& +\cdots+\frac{a_{n} b_{1} b_{2} \ldots b_{n-1}}{\lambda_{1}{ }^{n} \lambda_{2}{ }^{n}}\left(\lambda_{2}{ }^{n}-\lambda_{1}{ }^{n}\right)=0
\end{aligned}
$$

The equation (8) will be valid if, $\lambda_{1}=\lambda_{2}$, or contradictory to, the assumption stating. $\lambda_{1} \neq \lambda_{2}$. So the true is the positive eigenvalue of Leslie's matrix is singular, that is $\lambda_{1}=\lambda_{2}=b_{1}$.

(ii) Positive eigenvalues have a multiplicity of 1 $\lambda_{1} x_{4}=b_{3} x_{3}$

$x_{4}=\frac{b_{3}}{\lambda_{1}} x_{3} \rightarrow x_{4}=\frac{b_{3}}{\lambda_{1}}\left(\frac{b_{1} b_{2}}{\lambda_{1}{ }^{2}} x_{1}\right)$

$-b_{n-1} x_{n-1}+\lambda_{1} x_{n}=0$ 
$\lambda_{1} x_{n}=b_{n-1} x_{n-1}$

$x_{n}=\frac{b_{n-1}}{\lambda_{1}} x_{n-1}$

$x_{n}=\frac{b_{1} b_{2} \ldots b_{n-1}}{\lambda_{1}} x_{n-1}$

So obtained

$x_{2}=\frac{b_{1}}{\lambda_{1}} x_{1}$

$x_{3}=\frac{b_{2}}{\lambda_{1}} x_{2}=\frac{b_{1} b_{2}}{\lambda_{1}^{2}} x_{1}$

$x_{4}=\frac{b_{3}}{\lambda_{1}} x_{3}=\frac{b_{1} b_{2} b_{3}}{\lambda_{1}{ }^{3}} x_{1}$

$x_{n}=\frac{b_{1} b_{2} \ldots b_{n-1}}{\lambda_{1}} x_{n-1}=\frac{b_{1} b_{2} \ldots b_{n-1}}{\lambda_{1}{ }^{n-1}} x_{1}$

If for example taken $x_{1}=1$, then eigenvector $\mathbf{x}$ corresponds to $\lambda_{1}$ that is

$\mathbf{x}_{1}=\left[\begin{array}{c}x_{1} \\ x_{2} \\ x_{3} \\ x_{4} \\ \vdots \\ x_{n}\end{array}\right]=\left[\begin{array}{c}1 \\ b_{1} / \lambda_{1} \\ b_{1} b_{2} / \lambda_{1}{ }^{2} \\ b_{1} b_{2} b_{3} / \lambda_{1}{ }^{3} \\ \vdots \\ b_{1} b_{2} \ldots / \lambda_{1}{ }^{n-1}\end{array}\right]$

It is proven that Leslie's matrix eigenvect or of all elements is positive.

\section{Theorem 2}

If $\lambda_{1}$ is the unique positive eigenvalue of a Leslie matrix $L$ and if $\lambda_{i}$ is any real eigenvalues or complex of $L$, then $\left|\lambda_{k}\right| \leq \lambda_{1}$.

\section{Evidence:}

It has been proved that $\lambda_{1}$ is a single positive eigenvalue, then $\lambda_{1}$ is an eigenvalue that can be a real or complex number. If for example $\lambda_{k}=0$ it proves that $\left|\lambda_{k}\right| \leq$ $\lambda_{1}$. Then taken any $\lambda_{k}=r e^{i \theta}$ with, $i=\sqrt{-1}$, to prove the same as showing $|r| \leq \lambda_{1}$. The necessary conditions and sufficient conditions $\lambda_{k}$ to be an eigenvalue, $\lambda_{k}$ must be the solution of the system of equations

$$
\begin{aligned}
& q(\lambda)=\frac{a_{1}}{\lambda_{k}}+\frac{a_{2} b_{1}}{\lambda_{k}{ }^{2}}+\cdots+\frac{a_{n} b_{1} b_{2} \ldots b_{n-1}}{\lambda_{k}{ }^{n}}=1, \\
& q(\lambda)=1 \text { for } \lambda \neq 0 \\
& q(\lambda)=\frac{a_{1}}{r e^{i \theta}}+\frac{a_{2} b_{1}}{\left(r^{i \theta}\right)^{2}}+\cdots+\frac{a_{n} b_{1} b_{2} \ldots b_{n-1}}{\left(r^{i \theta}\right)^{n}}=1 \\
& \frac{a_{1}}{r}\left(e^{-i \theta}\right)+\frac{a_{2} b_{1}}{r^{2}}\left(e^{-2 i \theta}\right)+\cdots+\frac{a_{n} b_{1} b_{2} \ldots b_{n-1}}{r^{n}}\left(e^{-n i \theta}\right)=1 \\
& \frac{a_{1}}{r}(\cos (-\theta)+i \sin (-\theta))+\frac{a_{2} b_{1}}{r^{2}}(\cos (-2 \theta)(\ldots+ \\
& i \sin (-2 \theta))^{-2}+\cdots \\
& \ldots+\frac{a_{n} b_{1} b_{2} \ldots b_{n-1}}{r^{n}}(\cos (-n \theta)+i \sin (-n \theta))=1 \\
& \frac{a_{1}}{r}(\cos \theta-i \sin \theta)+\frac{a_{2} b_{1}}{r^{2}}(\cos 2 \theta-i \sin 2 \theta)++\cdots+ \\
& \frac{a_{n} b_{1} b_{2} \ldots b_{n-1}}{r^{n}}(\cos n \theta-i \sin n \theta)=1
\end{aligned}
$$

It means

$\frac{a_{1}}{r}(\cos \theta)+\frac{a_{2} b_{1}}{r^{2}}(\cos 2 \theta)+\cdots$

$\ldots+\frac{a_{n} b_{1} b_{2} \ldots b_{n-1}}{r^{n}}(\cos n \theta)=1$

And

$\frac{a_{1}}{r}(\sin \theta)+\frac{a_{2} b_{1}}{r^{2}}(\sin 2 \theta)+\cdots$

$\ldots+\frac{a_{n} b_{1} b_{2} \ldots b_{n-1}}{r^{n}}(\sin \theta)=0$

If taken only the real part is

$\frac{a_{1}}{r}(\cos \theta)+\frac{a_{2} b_{1}}{r^{2}}(\cos 2 \theta)+\cdots$

$\ldots+\frac{a_{n} b_{1} b_{2} \ldots b_{n-1}}{r^{n}}(\cos n \theta)=1$

Because $\lambda_{1}$ is an eigenvalue so $\lambda_{1}$ satisfies the equation $q(\lambda)=1$ so

$q(\lambda)=\frac{a_{1}}{\lambda_{1}}+\frac{a_{2} b_{1}}{\lambda_{1}{ }^{2}}+\cdots+\frac{a_{n} b_{1} b_{2} \ldots b_{n-1}}{\lambda_{1}{ }^{n}}=1$

If

$\frac{a_{1}}{r}(\cos \theta)+\frac{a_{2} b_{1}}{r^{2}}(\cos 2 \theta)+\cdots+\frac{a_{n} b_{1} b_{2} \ldots b_{n-1}}{r^{n}}(\cos n \theta)=$

$\frac{a_{1}}{\lambda_{1}}+\frac{a_{2} b_{1}}{\lambda_{1}^{2}}+\cdots+\frac{a_{n} b_{1} b_{2} \ldots b_{n-1}}{\lambda_{1}{ }^{n}}$

This equation is subtracted into

$a_{1}\left(\frac{\cos \theta}{r}-\frac{1}{\lambda_{1}}\right)+a_{2} b_{1}\left(\frac{\cos 2 \theta}{r^{2}}-\frac{1}{\lambda_{1}{ }^{2}}\right)+\cdots$

$\ldots+a_{n} b_{1} b_{2} \ldots b_{n-1}\left(\frac{\cos n \theta}{r^{n}}-\frac{1}{\lambda_{1}{ }^{n}}\right)=0$

$a_{1}\left(\frac{\lambda_{1} \cos \theta-r}{r \lambda_{1}}\right)+a_{2} b_{1}\left(\frac{\lambda_{1}{ }^{2} \cos 2 \theta-r^{2}}{r^{2} \lambda_{1}{ }^{2}}\right)+\cdots$

$\ldots+a_{n} b_{1} b_{2} \ldots b_{n-1}\left(\frac{\lambda_{1}^{n} \cos n \theta r^{n}}{r^{n} \lambda_{1}{ }^{n}}\right)=0$

Equations (9) apply if $\lambda_{1} \cos \theta-r=0$ Obtained $r=\lambda_{1} \cos \theta$. Because $-1 \leq \cos \theta \leq 1 \rightarrow-\lambda_{1} \leq r \leq$ $\lambda_{1}$, it means $|r| \leq \lambda_{1}$. If $\lambda_{1}$ meet $\left|\lambda_{k}\right|<\lambda_{1}$ is said that $\lambda_{1}$ is a dominant eigen value of $L$. Therefore the condition of theorem 2 is not strong enough to prove that the eigenvalues of Leslie's matrix are dominant.

\section{Theorem 3}

If two consecutive entries $a_{i}$ and $a_{i+1}$ in the first row of a Leslie matrix $L$ are not equal to zero then the positive eigenvalue of $L$ is dominant. It is called the dominant eigenvalue $\mathrm{j}$.

$\left|\lambda_{k}\right|<\lambda_{1}$ should not $\left|\lambda_{k}\right|=\lambda_{1}$ or indicated $\left|\lambda_{k}\right| \neq \lambda_{1}$. By definition $a_{i}>0$ and $a_{i+1}>0$. with $i=1,2, \ldots, n$,

Without prejudice generaly theorem be understood $i=$ 1. so $a_{1}>0$ and $a_{2}>0$, So it will be shown $\left|\lambda_{k}\right| \neq \lambda_{1}$ with $k=2,3, \ldots, n$, where $\lambda_{1}$ is positif. To prove it is done way of contradiction. That is, it will be assumed that $\lambda_{k}=\lambda_{1}$. If $\lambda_{k}=\lambda_{1}$ then $r e^{i \theta}=\lambda_{1}$, so $r(\cos \theta+$ $\sin \theta)=\lambda_{1} . \quad r \cos \theta+i r \sin \theta=\lambda_{1}+i 0 . \quad$ Obtained $r \cos \theta=\lambda_{1}$ and $r \sin \theta=0$. 
If the equation $r \cos \theta=\lambda_{1}$ multiplied by $\cos \theta$ be $r \cos ^{2} \theta=\lambda_{1} \cos \theta$. It means $\lambda_{1} \cos \theta \neq r$ or $\lambda_{1} \cos \theta-$ $r \neq 0$ based on the equation (9) that is

$$
\begin{aligned}
& a_{1}\left(\frac{\lambda_{1} \cos \theta-r}{r \lambda_{1}}\right)+a_{2} b_{1}\left(\frac{\lambda_{1}{ }^{2} \cos 2 \theta-r^{2}}{r^{2} \lambda_{1}{ }^{2}}\right)+\cdots \\
& \cdots+a_{n} b_{1} b_{2} \ldots b_{n-1}\left(\frac{\lambda_{1}{ }^{n} \cos n \theta-r^{n}}{r^{n} \lambda_{1}{ }^{n}}\right)=0
\end{aligned}
$$

Earned value $a_{1}=0$ and $a_{2}=0$. The statement is contrary to the assumption that states $a_{1}>0$ and $a_{2}>$ 0 . Meaning the wrong supposition $\left|\lambda_{k}\right| \neq \lambda_{1}$. In the next section it will always be assumed that the requirements of theorem 3 are met. It will be assumed that $L$ can be diagonalized. It is not necessary for the conclusions to be taken, but this will simplify the argument. In this case, $L$ has $n$ eigenvalues, $\lambda_{1}, \lambda_{2}, \ldots, \lambda_{n}$, which need not be different from each other, and $\mathrm{n}$ linearly independent eigenvectors $\mathbf{x}_{1}, \mathbf{x}_{2}, \ldots, \mathbf{x}_{n}$, which corresponds to the eigenvalue. In this list will be placed eigenvalues $\lambda_{1}$ the dominant first. A matrix $P$ will be formed whose columns are eigenvectors of $L$.

$P=\left[\mathbf{x}_{1}\left|\mathbf{x}_{2}\right| \mathbf{x}_{3}|\cdots| \mathbf{x}_{n}\right]$

$L P=L\left[\mathbf{x}_{1}\left|\mathbf{x}_{2}\right| \mathbf{x}_{3}|\cdots| \mathbf{x}_{n}\right]$

$=\left[L \mathbf{x}_{1}\left|L \mathbf{x}_{2}\right| L \mathbf{x}_{3}|\cdots| L \mathbf{x}_{n}\right]$

$=\left[\lambda_{1} \mathbf{x}_{1}\left|\lambda_{2} \mathbf{x}_{2}\right| \lambda_{3} \mathbf{x}_{3}|\cdots| \lambda_{n} \mathbf{x}_{n}\right]$

$=\left[\mathbf{x}_{1}\left|\mathbf{x}_{2}\right| \mathbf{x}_{3}|\cdots| \mathbf{x}_{n}\right]\left[\begin{array}{cccc}\lambda_{1} & 0 & \cdots & 0 \\ 0 & \lambda_{2} & \cdots & 0 \\ \vdots & \vdots & & \vdots \\ 0 & 0 & \cdots & \lambda_{n}\end{array}\right]=P D$

Because column vectors of the linear matrices $P$ are linearly independent, and $P$ is reversible so that the matrix $L$ can be diagonalized. The diagonalization of $L$ will be given by the equation

$L=P D P^{-1}$

$L=P\left[\begin{array}{cccc}\lambda_{1} & 0 & \cdots & 0 \\ 0 & \lambda_{2} & \cdots & 0 \\ \vdots & \vdots & & \vdots \\ 0 & 0 & \cdots & \lambda_{n}\end{array}\right] P^{-1}$

From this equation is obtained

$L^{k}=P\left[\begin{array}{cccc}\lambda_{1}^{k} & 0 & \cdots & 0 \\ 0 & \lambda_{2}^{k} & \cdots & 0 \\ \vdots & \vdots & & \vdots \\ 0 & 0 & \cdots & \lambda_{n}^{k}\end{array}\right] P^{-1}$

for $k=1,2, \ldots$

For any vector of age distribution it will first $\mathbf{x}^{(0)}$ be obtained

$L^{k} \mathbf{X}^{(0)}=P\left[\begin{array}{cccc}\lambda_{1}^{k} & 0 & \cdots & 0 \\ 0 & \lambda_{2}^{k} & \cdots & 0 \\ \vdots & \vdots & & \vdots \\ 0 & 0 & \cdots & \lambda_{n}^{k}\end{array}\right] P^{-1} \mathbf{X}^{(0)}$

for $k=1,2, \ldots$
By dividing two sections of this equation by $\lambda_{1}^{k}$ and using the fact that $\mathbf{x}^{(k)}=L^{(k)} \mathbf{x}^{(0)}$, it will be obtained

$\frac{1}{\lambda_{1}^{k}} \mathbf{x}^{(k)}=P\left[\begin{array}{cccc}1 & 0 & \cdots & 0 \\ 0 & \left(\lambda_{2} / \lambda_{1}\right)^{k} & \cdots & 0 \\ \vdots & \vdots & & \vdots \\ 0 & 0 & \cdots & \left(\lambda_{n} / \lambda_{1}\right)^{k}\end{array}\right] P^{-1} \mathbf{x}^{(0)}(10)$

Because $\lambda_{1}$ is the dominant eigen value that is $\left|\lambda_{1}\right|>$ $\left|\lambda_{i}\right|$, then $\left|\lambda_{i} / \lambda_{1}\right|<1$ for $i=2,3, \ldots, n$. It is clear that $\left(\lambda_{i} / \lambda_{1}\right)^{k} \rightarrow 0$ if $k \rightarrow \infty$ for $i=2,3, \ldots, n$.

Using this fact, it can take limits from both sides of (10) to get

$\lim _{k \rightarrow \infty}\left\{\frac{1}{\lambda_{1}^{k}} \mathbf{x}^{(k)}\right\}=p\left[\begin{array}{ccccc}1 & 0 & 0 & \cdots & 0 \\ 0 & 0 & 0 & \cdots & 0 \\ \vdots & \vdots & \vdots & & \vdots \\ 0 & 0 & 0 & \cdots & 0\end{array}\right] P^{-1} \mathbf{x}^{(\mathbf{0})}$

It will then be declared the first entry of the column vector $P^{-1} \mathbf{x}^{(\mathbf{0})}$ with the constants $c$, then

$P^{-1} \mathbf{x}^{(\mathbf{0})}=\left[\begin{array}{c}c \\ c_{1} \\ \vdots \\ c_{n-1}\end{array}\right]$.

This result is entered into the equation (11)

$\lim _{k \rightarrow \infty}\left\{\frac{1}{\lambda_{1}^{k}} \mathbf{x}^{(k)}\right\}=p\left[\begin{array}{ccccc}1 & 0 & 0 & \cdots & 0 \\ 0 & 0 & 0 & \cdots & 0 \\ \vdots & \vdots & \vdots & & \vdots \\ 0 & 0 & 0 & \cdots & 0\end{array}\right]\left[\begin{array}{c}c \\ c_{1} \\ \vdots \\ c_{n-1}\end{array}\right]=$

$\left[\mathbf{x}_{1}\left|\mathbf{x}_{2}\right| \mathbf{x}_{3}|\cdots| \mathbf{x}_{n}\right]$

$\left[\begin{array}{ccccc}1 & 0 & 0 & \cdots & 0 \\ 0 & 0 & 0 & \cdots & 0 \\ \vdots & \vdots & \vdots & & \vdots \\ 0 & 0 & 0 & \cdots & 0\end{array}\right]\left[\begin{array}{c}c \\ c_{1} \\ \vdots \\ c_{n-1}\end{array}\right]=\left[\mathbf{x}_{1}\left|\mathbf{x}_{2}\right| \mathbf{x}_{3}|\cdots| \mathbf{x}_{n}\right]\left[\begin{array}{c}c \\ 0 \\ \vdots \\ 0\end{array}\right]$

$=c \mathbf{x}_{1}$

So that the right-hand side of (11) can be written as $c \mathbf{x}_{\mathbf{1}}$, where $c$ is a positive controw that depends only on the vector of age distribution first $\mathbf{x}^{(0)}$.So (11) be

$\lim _{k \rightarrow \infty}\left\{\frac{1}{\lambda_{1}^{k}} \mathbf{x}^{(k)}\right\}=c \mathbf{x}_{1}$

The equation (12) gives an approximation

$\mathbf{x}^{(k)} \sim c \lambda_{1}^{k} \mathbf{x}_{\mathbf{1}}$

For large k values. From (13) also obtain

$\mathbf{x}^{(k-1)} \simeq c \lambda_{1}^{k-1} \mathbf{x}_{1}$

By comparing equations (13) and equations (14) obtained 
$\mathbf{x}^{(k)} \simeq \lambda_{1} \mathbf{x}^{(k-1)}$

For large $k$ values. This means that for large time values each age distribution vector is a scalar multiple of the previous age distribution vector, and the scalar is the positive eigenvalue of Leslie's matrix. Consequently, the proportion of females in each group of age groups will be constant.Then we will review the equations (13) that give the age distribution vector of the population for a long time:

$\mathbf{x}^{(k)} \simeq c \lambda_{1}^{k} \mathbf{x}_{1}$

Three cases will appear in accordance with eigenvalues $\lambda_{1}$ : (i) The total population will eventually tend to increase/increase if $\lambda_{1}>1$, (ii) The total population will eventually tend to decrease/decrease if $\lambda_{1}<1$, (iii) Population will tend to be stable/fixed if $\lambda_{1}=1$. If the population tends to decline then it can be said also that the population growth rate is negative, where as if the population number increases it can be said also that the population growth rate is positive.

\section{Data on Population of Women and Number of Children Born in 2010 and Year 2015 in Trenggalek Regency}

The following data on the number of female population from 2010-2015 in Trenggalek Regency that has been studied in the research. Because only a few women over 45 years old who gave birth to a child, it will be limited from the population of women aged between 15 and 49 years this is due to the age of female fertility interval of 15-49 years. This data is for the age group with the period of 5 years, so the total number is 14 age groups. From Table 3 data will be analyzed by looking for parameter $a_{i}$ (fertility number) and $b_{i}$ parameter (life expectancy). Leslie Matrix Model can be used to determine the number of female population the next 6 years. Using Leslie's matrix, according to Table 4. The female population is divided into several age-class intervals, with the interval of female fertility 15-49 years. To calculate the number of female population by matrix method Leslie influenced by fertility rate $\left(a_{i}\right)$ life expectancy $\left(b_{i}\right)$. Here is a settlement step to predict the number and growth rate in Trenggalek Regency in 2021.

Table 3. Number of female population year 2010-2015.

\begin{tabular}{lllll}
\hline Age Class & $\begin{array}{l}\text { Number } \\
\text { of Female } \\
\mathbf{2 0 1 0}\end{array}$ & $\begin{array}{l}\text { Number } \\
\text { of Female } \\
\mathbf{2 0 1 5}\end{array}$ & ai & bi \\
\hline $0-4$ & 24,284 & 22,860 & 0 & 0.978257 \\
$5-9$ & 24,529 & 23,756 & 0 & 1.019365 \\
$10-14$ & 26,129 & 25,004 & 0 & 0.933752 \\
$15-19$ & 24,704 & 24,398 & 0.0099 & 0.903538 \\
$20-24$ & 22,592 & 22,321 & 0.2599 & 1.018900 \\
$25-29$ & 24,790 & 23,019 & 0.2688 & 0.943687 \\
$30-34$ & 24,627 & 23,394 & 0.2698 & 1.055955 \\
$35-39$ & 26,632 & 26,005 & 0.2599 & 1.052418 \\
$40-44$ & 28,185 & 28,028 & 0.0795 & 0.969700 \\
$45-49$ & 25,671 & 27,331 & 0.0374 & 0.994313 \\
$50-54$ & 21,950 & 25,525 & 0 & 0.987198 \\
$55-59$ & 17,401 & 21,669 & 0 & 0.925694 \\
$60-64$ & 13,651 & 16,108 & 0 & 2.738627 \\
$65+$ & 35,320 & 37,385 & 0 & 0 \\
Total & 340,465 & 346,803 & & \\
\hline
\end{tabular}

Next will be analyzed using the MAPLE 16 program to determine the dynamics of the growth process based on the eigenvalues and eigenvectors of Leslie matrix. Then using the equation approach for the age limited distribution $\mathbf{x}^{(k)} \simeq \lambda_{1} \mathbf{x}^{(k-1)} \sim$ will find the rate growth of female population in Trenggalek Regency.

Having formed Leslie's matrix, based on equation (3), that is $\mathbf{x}^{(k)}=L \mathbf{x}^{(k-1)}$ with $k=1,2, \ldots$ to predict the number of women in 2021. Based on Table. 3 we obtain the following Leslie matrix $\mathbf{x}^{(k)}=L \mathbf{x}^{(k-1)}=$

$\left[\begin{array}{ccccccccccccccc}0 & 0 & 0 & 0.0099 & 0.2599 & 0.2688 & 0.2698 & 0.2599 & 0.0795 & 0.0374 & 0 & 0 & 0 & 0 \\ 0.9782 & 0 & 0 & 0 & 0 & 0 & 0 & 0 & 0 & 0 & 0 & 0 & 0 & 0 \\ 0 & 1.0193 & 0 & 0 & 0 & 0 & 0 & 0 & 0 & 0 & 0 & 0 & 0 & 0 \\ 0 & 0 & 0.9337 & 0 & 0 & 0 & 0 & 0 & 0 & 0 & 0 & 0 & 0 & 0 \\ 0 & 0 & 0 & 0.9035 & 0 & 0 & 0 & 0 & 0 & 0 & 0 & 0 & 0 & 0 \\ 0 & 0 & 0 & 0 & 1.0189 & 0 & 0 & 0 & 0 & 0 & 0 & 0 & 0 & 0 \\ 0 & 0 & 0 & 0 & 0 & 0.9436 & 0 & 0 & 0 & 0 & 0 & 0 & 0 & 0 \\ 0 & 0 & 0 & 0 & 0 & 0 & 1.0559 & 0 & 0 & 0 & 0 & 0 & 0 & 0 \\ 0 & 0 & 0 & 0 & 0 & 0 & 0 & 1.0524 & 0 & 0 & 0 & 0 & 0 & 0 \\ 0 & 0 & 0 & 0 & 0 & 0 & 0 & 0 & 0.9697 & 0 & 0 & 0 & 0 & 0 \\ 0 & 0 & 0 & 0 & 0 & 0 & 0 & 0 & 0 & 0.9943 & 0 & 0 & 0 & 0 \\ 0 & 0 & 0 & 0 & 0 & 0 & 0 & 0 & 0 & 0 & 0.9871 & 0 & 0 & 0 \\ 0 & 0 & 0 & 0 & 0 & 0 & 0 & 0 & 0 & 0 & 0 & 0.9256 & 0 & 0 \\ 0 & 0 & 0 & 0 & 0 & 0 & 0 & 0 & 0 & 0 & 0 & 0 & 2.7386 & 0\end{array}\right]\left[\begin{array}{c}22,860 \\ 23,756 \\ 25,004 \\ 24,398 \\ 23,019 \\ 23,394 \\ 26,005 \\ 28,028 \\ 25,331 \\ 25,525 \\ 21,669 \\ 16,108 \\ 37,385\end{array}\right]=\left[\begin{array}{c}28,553 \\ 22,361 \\ 24,216 \\ 23,347 \\ 22,044 \\ 22,742 \\ 21,722 \\ 24,702 \\ 27,368 \\ 27.178 \\ 27,175 \\ 25,198 \\ 20,058 \\ 39.343\end{array}\right]$


Then by using the equation approach for limited age distribution: $\quad \mathbf{x}^{(k)} \simeq \lambda_{1} \mathbf{x}^{(k-1)} \quad$ so $\quad \mathbf{x}^{(k)} \simeq$ $1,000391 \mathbf{x}^{(k-1)}$. From the eigen value obtained $\lambda_{1}>1$ so that every five years of female population in Trenggalek Regency will tend to increase or in other words growth rate of woman population in Trenggalek Regency tends to be positive value.

Table 4. Number of women year 2010 and 2015 \& women's prediction year 2021.

\begin{tabular}{llll}
\hline Age Class & $\begin{array}{l}\text { Number of } \\
\text { Female 2010 }\end{array}$ & $\begin{array}{l}\text { Number of } \\
\text { Female 2015 }\end{array}$ & $\begin{array}{l}\text { Number of } \\
\text { Female 2021 }\end{array}$ \\
\hline $0-4$ & 24,284 & 22,860 & 28,553 \\
$5-9$ & 24,529 & 23,756 & 22,361 \\
$10-14$ & 26,129 & 25,004 & 24,216 \\
$15-19$ & 24,704 & 24,398 & 23,347 \\
$20-24$ & 22,592 & 22,321 & 22,044 \\
$25-29$ & 24,790 & 23,019 & 22,742 \\
$30-34$ & 24,627 & 23,394 & 21,722 \\
$35-39$ & 26,632 & 26,005 & 24,702 \\
$40-44$ & 28,185 & 28,028 & 27,368 \\
$45-49$ & 25,671 & 27,331 & 27,178 \\
$50-54$ & 21,950 & 25,525 & 27,175 \\
$55-59$ & 17,401 & 21,669 & 25,198 \\
$60-64$ & 13,651 & 16,108 & 20,058 \\
$65+$ & 35,320 & 37,385 & 39,343 \\
Total & $\mathbf{3 4 0 , 4 6 5}$ & $\mathbf{3 4 6 , 8 0 3}$ & $\mathbf{3 5 6 , 0 0 7}$ \\
\hline
\end{tabular}

\section{CONCLUSION}

Based on the data analysis of Leslie Matrix in female population in Trenggalek Regency, it can be concluded that eigen value for the total population of women in Trenggalek regency in year 2021 amounted to 356.007 .
Distribution age limit for female population in Trengggalek Regency is $\mathbf{x}^{(k)} \simeq \lambda_{1} \mathbf{x}^{(k-1)}$ with positive eigen value $\lambda_{1}>1$ is greater than one that is equal to 1,000391 so that the number of female population in Trenggalek Regency in year 2021 tend to increase or can be said growth rate of population tends to be positive value.

\section{REFERENCES}

Adiwijaya. 2014. Application of matrices and vector spaces. Yogyakarta: Graha Ilmu.

Anton, H. \& Rorres, C. 1988. Application of linear algebra. Jakarta: Erland.

Anton, H \& Rorres, C. 2004. Linear elementary algebra (app version). Jakarta: Erland.

Central Bureau of Statistics. 2013. Projection of Indonesian population (Indonesian population projection) 2010-2035, Jakarta.

Corazon, Nurul, H and Yusianta, M, 2016. Leslie's matrix application to predict the number and growth rate of women in Riau Province by 2017. Journal of Mathematical and Statistical Science (JSMS), 2 (3), 1-11, ejournal.uinsuska.ac.id/index.php/JSMS/article / download / 3098 /

Iswanto, R.J. 2012. Applied and applied mathematical modeling. Yogyakarta: Graha Ilmu.

Kariadinata, R. 2013. Algebra of elementary matrices. Bandung: CV Pustaka Setia.

Pratama, Prihandono and Kusumastuti 2013. Leslie's matrix application to predict the number and rate of growth of a population. Bimaster: Math Scientific Bulletin. Stat. And Applied, $\quad 2 \quad$ (3), 163-172. http://jurnal.untan.ac.id/index.php/jbmstr/article/view/3859.

Tarumingkeng, R.C. 1994, Population Dynamics (Quantitative Ecological Studies), Pustaka Sinar Harapan, Jakarta 
THIS PAGE INTENTIONALLY LEFT BLANK 